\title{
PULSARS AND SOLAR-SYSTEM EPHEMERIDES
}

\author{
J.F. CHANDLER \\ Harvard/Smithsonian Center for Astrophysics \\ 60 Garden Street, Cambridge, MA 02138
}

\begin{abstract}
The analysis of pulsar time-of-arrival data is intimately bound up with planetary ephemerides. Highly accurate ephemerides are required for Earth and Moon and, to a lesser degree, for the other planets, in order to make full use of the timing data for millisecond-class pulsars. These data, in turn, present an opportunity for improving planetary ephemerides in a variety of ways. Fitting the Earth and Moon orbital parameters to the timing data is the obvious first step, though it is less valuable in the short term for many applications than using the current accumulation of spacecraft-tracking and lunar laser ranging data. By themselves, the pulsar timing data convey no information on the orientation of Earth's orbit, since each pulsar's position on the sky must be determined from those same data. However, independent pulsar position measurements by VLBI, in combination with the timing-derived positions, can serve to fix the orientation of Earth's orbit with respect to the radio reference frame and thereby link the planetary and radio frames. In the long run, the acquisition of timing data over increasing time spans and with improving precision should prove to be an important factor in determining the shape, as well as the orientation, of Earth's orbit. In addition, pulsar timing over a sufficiently long span can directly measure a planet mass through the reaction of the rest of the solar system. The effect must be observed for a major fraction of the orbital period of the planet in question so that the signature can be separated from that of the ordinary spin-down of each pulsar. Finally, pulsar timing can be used to probe gravitational physics, a field with far-reaching consequences and a basic part of the framework for constructing the ephemerides.
\end{abstract}




\section{Introduction}

In this paper, I discuss contributions of pulsar timing to the development of planetary ephemerides and vice versa. Pulsars, especially millisecond-class pulsars, present a unique opportunity to track the motion of Earth while nominally observing a distant astronomical object. Although the accepted mechanisms for pulsed emission have not been confirmed by direct observation, the extreme regularity of the pulses has been abundantly demonstrated over years of timing observations. Any object that emits signals regularly is, in effect, a clock and is potentially useful as a standard for comparison with other clocks. Even "young" pulsars, which are characterized by large pulse period derivatives and occasional timing "glitches" attributed to the sudden relaxation of rigid structures stressed by changing spin rates, generally show regular behavior between glitches. Unfortunately, the unpredictability of such behavior in young pulsars renders them useless for long-term comparisons.

Millisecond pulsars, however, have not only shorter periods than ordinary pulsars, but also slower spin-down rates and an apparent lack of glitches. These characteristics place them among the best clocks known to us in the Universe and give them the potential for high-precision measurements of Earth motion.

\section{Modeling pulse arrival times}

In order to account for pulsar timing observations, it is necessary to deal simultaneously with properties of both the pulsar and the observer. Let us first consider the train of pulses emitted by the pulsar. Although individual pulses display considerable variability in amplitude and even in shape, it is possible for the observer to form an average pulse profile by integrating over time spans as short as a few minutes and to determine the arrival time of a reference pulse within each span. Such averaging yields a timing signal characterized by remarkably few parameters. The model of pulse emission must include the pulse period and period derivatives, as well as an epoch for the arrival of one particular pulse (or, equivalently, an initial pulse phase and the first few time derivatives of the phase). These are the only required parameters intrinsic to the pulsar. In addition, of course, the pulsar's position on the sky and proper motion must be included because the observing platform is moving. For a nearby pulsar, even the distance must be included in calculating the annual variations in pulsar-Earth path length; the approximation of projecting the Sun-Earth vector onto the mean line of sight is not sufficiently accurate. The distance is also important in the sense that interstellar dispersion of the pulsar signals increases with distance. Indeed, the dispersion is one of the most striking features of pulsar signals, since it 
must be determined before the pulsing can be detected in the first place. If the pulsar has a known binary companion, the (time-variable) orbital elements must also be included. However, the fastest known pulsar (and the best for the purposes of solar-system dynamics) is PSR B1937+21, a solitary object.

These few parameters adequately describe the emitted pulse train. For the intrinsic pulsar parameters, there is no need to assign a physical significance, nor (in the absence of a binary companion) any real interest from the point of view of solar-system dynamics. They are simply numbers to be determined. Similarly, the interstellar dispersion, once known well enough to permit detection of the pulses, is nothing more than a time-variable calibration factor that can be readily determined from dual-frequency observations of the pulsar signals.

We turn, then, to the effects of the solar system on pulse arrival times seen by a terrestrial observer. The most obvious is the annual variation due to Earth's orbit around the Sun, but there are many others, and pulsar timing depends critically on a detailed and accurate picture of these effects. If planetary ephemerides did not already exist, they would need to be devised for and from the timing of pulsar signals. In fact, the process would be analogous to that of detecting and characterizing planets in orbit around a pulsar, except that our own planets affect the signals from every pulsar at once and are therefore much more easily characterized. In any event, planetary ephemerides $d o$ exist, and pulse timing interpretation can take advantage of them in describing the four-dimensional geometry of the observer. Table 1 shows a summary of the effects important for that description.

Most, but not all, of these effects are simply variable displacements, like that of an observatory from Earth's center, of Earth from the EarthMoon barycenter, and so on. The leap second, of course, is a purely human artifact that affects only the time scale and not the pulse arrival times themselves. Similarly, the item called "local proper time" is simply the variation in rate of Earth-borne clocks in a relativistic sense, due to the changing velocity and gravitational potential. The items marked "orbit" (aside from Earth and Moon) do not directly affect the pulse arrival times, since they are the offsets of the Sun from the solar-system barycenter, but they serve as markers for the corresponding perturbations on Earth's orbit. In a numerically integrated planetary ephemeris, such as those used for the analysis of pulsar data, these perturbations are not presented as separate terms, but are simply included in the Earth orbit. The amplitude of the Shapiro delay in the table is calculated for a "typical" pulsar; it would be larger for a pulsar close to the ecliptic. 
TABLE 1. Ephemeris contributions to pulse timing variation

\begin{tabular}{llr}
\hline Term & Period & $\begin{array}{r}\log (\text { amplitude) } \\
(\mathrm{sec})\end{array}$ \\
\hline Earth orbit & $1 \mathrm{yr}$ & 3 \\
Earth eccentricity & $1 \mathrm{yr}$ & 1 \\
Jupiter orbit & $12 \mathrm{yr}$ & 0 \\
Saturn orbit & $29 \mathrm{yr}$ & 0 \\
Leap seconds & $1-2 \mathrm{yr}$ & 0 \\
Neptune orbit & $165 \mathrm{yr}$ & 0 \\
Uranus orbit & $84 \mathrm{yr}$ & -1 \\
Earth rotation & $1 \mathrm{day}$ & -2 \\
Earth precession & long & -2 \\
Moon orbit & $27 \mathrm{days}$ & -2 \\
Earth proper time & $1 \mathrm{yr}$ & -3 \\
Venus orbit & $0.6 \mathrm{yr}$ & -3 \\
Pluto orbit & $248 \mathrm{yr}$ & -4 \\
Mars orbit & $2 \mathrm{yr}$ & -4 \\
Mercury orbit & $0.2 \mathrm{yr}$ & -5 \\
Shapiro delay & $1 \mathrm{yr}$ & -5 \\
Earth nutation & $19 \mathrm{yr}$ & -6 \\
Local proper time & $1 \mathrm{day}$ & -6 \\
Ceres orbit & $5 \mathrm{yr}$ & -6 \\
\hline
\end{tabular}

\section{Ephemeris frames}

One result of the use of pre-computed planetary ephemerides for the analysis of pulse timing data, as opposed to a combined analysis of pulsar and planetary data, is the dependence of the final results upon the choice of ephemeris. Such a dependence would be a serious drawback if the differences induced by switching from one ephemeris to another produced significant changes in interpretation. Fortunately, the ephemerides used for pulse timing are nearly interchangeable, aside from offsets in the orientation and mean motion of Earth's orbit. Since the annual signature of Earth motion dominates the variations of pulse arrival times (see Table 1), each ephemeris defines, in effect, its own reference frame via the specification of coordinates of the Earth-Moon barycenter as a function of time. That reference frame then provides the context for the pulsar positions and proper motions obtained using that ephemeris.

We can confirm the near-equivalence of separate ephemerides by direct comparison of the coordinates. Obviously, these coordinates differ system- 
atically, and the differences can largely be described by a simple, linearly varying orientation offset between the corresponding reference frames. In practice, the residual differences, though still systematic, can be neglected or treated merely as ephemeris "noise" because they are so small.

Among the ephemerides used for pulse timing are a set from MIT and more recently from the Center for Astrophysics: PEP311 (1969), PEP740 (1984), and PEP740R (also 1984). The latter was produced, solely for the convenience of pulsar observers, to agree with the orientation of PEP311 at epoch 1982.9 , by rotating all the coordinates by a fixed amount. Another series of ephemerides has been distributed from JPL, including DE96, DE118, and DE200 (see Standish 1982). The latter is the only one of these that is nominally aligned to the equator and equinox of J2000; the others are aligned to B1950. That discrepancy is merely one more offset of orientation and therefore makes no difference to the effective interchangeability of ephemerides.

The ephemerides were compared directly, one to another, using a linearized least-squares fit of tabulations of the cartesian coordinates at fourday intervals over a span of twelve years. The process involved solving for an overall scale factor, an offset of orientation, and a steady rotation. The solutions for these parameters are not intrinsically interesting, except insofar as they indicate the transformation from one ephemeris frame to another. The key point is the postfit RMS residual deviation between the two ephemerides after applying the transformation. In all cases, i.e., for the ephemerides mentioned above, considered pairwise, this was about $10^{-10}$ AU. A further check on this technique was a series of one-year fits covering the twelve-year span for each pair of ephemerides solving only for the offset of orientation. The resulting set of angular offsets was consistent with the offset and rotation rate determined by the overall fit, to within the indicated "noise" level. For further discussion of this fitting procedure and the resulting transformations, see Bartel et al. (1996).

\section{Applications of pulse timing}

In the near future, pulse timing analysis is not competitive with the more conventional techniques for refining solar-system ephemerides. Even the best available millisecond pulsar, PSR B1937+21, displays timing noise on the order of $0.3 \mu \mathrm{s}=100 \mathrm{~m}$ (Kaspi et al. 1994). By comparison, the set of spacecraft-tracking data from interplanetary missions includes range measurements between Earth and Mars with an accuracy of about $10 \mathrm{~m}$ (the Viking landers). Further, the lunar laser ranging data have reached an accuracy of a few $\mathrm{cm}$ between laser stations on Earth and the retroreflectors on the Moon. These lunar data, being all Earth-based, are much less sen- 
sitive to the orientation of the lunar orbit than to its shape, but they have the advantage over pulse timing data of sensing the Moon directly, rather than through the reaction of Earth. Pulse timing can therefore make no significant contributions in the foreseeable future to the Moon ephemeris. Potentially, the long-term accumulation of pulse timing data for more and better pulsars could shift the balance away from the spacecraft-based data now available, but the latter remain for now the primary determinant for the Earth ephemeris.

The one contribution pulsars are uniquely equipped to make is in the overall orientation of the planetary ephemerides. This orientation is only weakly determined by the conventional high-precision techniques, which rely on topocentric distance measurements for objects all in the same system. Though the spinning Earth provides an inertial reference, these techniques tie that reference to the planetary system only by triangulation over terrestrial baselines. Pulse timing measurements, on the other hand, can make use of baselines across Earth's orbit, and the angular accuracy for a $100 \mathrm{~m}$ distance resolution is therefore better than 1 mas. However, the position of each pulsar on the sky is customarily determined from the pulse timing, and it is necessary to establish the pulsar position independently before the timing data can be used to set the orientation of the ephemerides. The technique of Very Long Baseline Interferometry (VLBI) is well suited to the task of measuring pulsar positions with respect to an independent reference frame, namely, that of the extragalactic radio sources. PSR B1937+21 has been determined with respect to that frame with an accuracy of about 3 mas (Bartel et al. 1990 and 1996). The combination of VLBI and pulse timing, then, can relate the planetary ephemeris frame to the radio reference frame to about that level.

Another possible application of pulse timing is the determination of planet masses. Such a determination is sensitive to correlations between the pulsar model and planet orbital elements, and requires a long time base to break degeneracies, but it shows promise for some of the planets. Table 2 gives the results of a case study assuming 20 years of timing data twice per month with $0.3 \mu$ s uncertainty, along with the current state of knowledge for planet orbital elements, and ignoring uncertainties in pulsar spin-down, etc. For comparison, the table also shows the current uncertainties in the planet masses, from a combination of spacecraft and planetary data. For the terrestrial planets, even 20 years of pulse timing is insufficient to match the accuracy of existing techniques, and 20 years is also too short a time span for the planets outward of Saturn, but Jupiter and Saturn both fall into the intermediate range. Certainly, the results of the Galileo mission are expected to improve the uncertainty in Jupiter's mass, but Saturn remains a possible target for improvement. 
TABLE 2. Planet mass uncertainties in solar mass units

\begin{tabular}{lrr}
\hline Planet & $\begin{array}{r}\text { Current } \\
\text { uncertainty }\end{array}$ & $\begin{array}{r}\text { Possible uncertainty } \\
\text { from pulse timing } \\
\text { over 20 years } \\
\end{array}$ \\
& $(\log )$ & -10.0 \\
\hline Mercury & -11.0 & -10.0 \\
Venus & -11.7 & -10.5 \\
Mars & -11.5 & -10.7 \\
Jupiter & -9.3 & -11.1 \\
Saturn & -8.2 & \\
\hline
\end{tabular}

Finally, pulse timing can be used to study gravitational physics through a variety of effects. As shown in Table 1, the Shapiro delay due to the Sun's gravitational potential plays a role in pulse timing residuals. In order to make a useful contribution, this effect must be magnified by the discovery of a suitable pulsar near the ecliptic, so that observations of the delay near conjunction could be made, and, even there, this technique would still have to compete with the corresponding spacecraft experiments (primarily the Viking mission to Mars). The Shapiro delay due to propagation of pulsar signals in a binary system or the effect of gravitational radiation in such a system (Taylor and Weisberg 1982), or even the possible timing variations due to background gravitational radiation (see, for example, Davis et al. 1985), may also be useful, but these are not directly relevant to solarsystem dynamics. An interesting possibility is the use of pulse timing, in combination with VLBI measurements, to study the gravitational redshift of Earth-borne clocks (Shapiro 1986, Chandler 1990). This effect has already been confirmed to a fractional accuracy of $10^{-4}$ within the potential well of Earth (Vessot et al. 1980), but nothing approaching that accuracy has been achieved in the solar potential. One or two suitably placed millisecond pulsars whose positions can be measured by VLBI to the level of 1 mas would yield a fractional redshift test of $10^{-3}$.

Thus, we see that solar-system dynamics and pulse timing serve each other in several ways. Highly accurate planetary ephemerides are essential to the proper modeling of pulse arrival times. Pulse timing, in turn, offers an opportunity to determine the orientation of the planetary ephemeris frame, refine some of the parameters in the solar-system model, and even to test the underlying gravitational physics. 


\section{Acknowledgments}

This work was supported in part by NASA under grant NAGW-3666. Travel funds for presenting this paper at IAU Symposium 172 were supplied through the Organizing Committee.

\section{References}

Bartel, N., Cappallo, R., Whitney, A., Chandler, J., Ratner, M., Shapiro, I., and Tang, G. 1990, in Workshop on Impact of Pulsar Timing on Relativity and Cosmology (Berkeley).

Bartel, N., Ebbers, A., Pan, R., Cappallo, R.J., Chandler, J.F., Ratner, M.I., and Shapiro, I.I. 1996, in preparation.

Chandler, J.F. 1990, in Workshop on Impact of Pulsar Timing on Relativity and Cosmology (Berkeley).

Davis, M.M., Taylor, J.H., Weisberg, J.M., and Backer, D.C. 1985, Nature, 315, 547.

Kaspi, V.M., Taylor, J.H., and Ryba, M. 1994, Ap. J., 428, 713.

Shapiro, I. 1986, in Proceedings of the Workshop on the Arecibo Upgrade (Cornell).

Standish, E.M. 1982, $A \& B$, 114, 297.

Taylor, J.H. and Weisberg, J.M. 1982, Ap. J., 235, 908.

Vessot, R.F.C., et al. 1980, Phys. Rev. Lett., 45, 2081. 